\title{
THE NORMALITY OF TIME-INVARIANT, SUBORDINATIVE OPERATORS IN A HILBERT SPACE
}

\author{
BY P. MASANI
}

Communicated by P. R. Halmos, December 7, 1964

1. Introduction. Let $\left(U_{t}, t \in R\right)$ be a strongly continuous group of unitary operators on a (complex) Hilbert space $\mathfrak{H C}$ onto $\mathfrak{H C}$, and $E$ be its spectral measure on the family $B$ of Borel subsets of the real number field $R$, so that $U_{t}=\int_{-\infty}^{\infty} e^{i \lambda t} E(d \lambda)$ (Stone's Theorem). Let $\delta_{x}$ be the cyclic subspace generated by $x$ under the action of the $U_{t}, t \in R$, or, equivalently, under that of $E(B), B \in \beta$. Let $L_{x}$ be the cyclic projection of $x$, i.e., the projection on $\mathfrak{H C}$ with range $\delta_{x}$. Using a term due to Kolmogorov $[2, \S 4]$ we shall say that $x$ is subordinate to $y$ if $S_{x} \subseteq S_{y}$.

Our purpose is to assert the following theorem and deduce some corollaries which generalize known results:

1.1. THEOREM. Given a strongly continuous unitary group $\left(U_{t}, t \in R\right)$ with spectral measure $E$ on the family $B$ of Borel subsets of $R$, let $T$ be any (single-valued, unbounded) linear operator from 30 to $\mathfrak{B C}$ such that

(i) $U_{t} T=T U_{t}$ for all $t \in R(T$ is "time-invariant"),

(ii) $T(x) \in \mathcal{S}_{x}$ for all $x \in D_{T}(T$ is "subordinative"),

(iii) $T$ is closed and $\mathfrak{H C}_{0}=$ clos $\mathscr{D}_{T}$ is separable.

Then there exists a complex-valued Borel-measurable function $\phi$ on $R$ such that

$$
T=\int_{-\infty}^{\infty} \phi(\lambda) E_{0}(d \lambda), \text { where } E_{0}=\text { Rstr. } \mathcal{F}_{0} E .^{2}
$$

$\phi$ is unique up to sets of zero $E_{0}$-measure.

2. Statistical theory of linear filters. Theorem 1.1 has its genesis in the statistical theory of linear filters as conceived by N. Wiener. In this theory the signals are realizations of strictly stationary stochastic processes (S.P.). It is assumed that these processes are governed by a single measure-preserving, ergodic flow over a probability space $(\Omega, B, P)$. This flow induces the unitary group $\left(U_{t}, t \in R\right)$ on the Hilbert space $H=L_{2}(\Omega, B, P) . T$ is the filter transformation; it

1 This work was supported by the Office of Naval Research, and was begun at the Bell Telephone Laboratories, Murray Hill, New Jersey.

${ }^{2}$ Rstr. $D A$ denotes the restriction of the operator $A$ to the subset $D$ of its domain. 
converts the random function $f$ of the input S.P. $\left(U_{t}(f), t \in R\right)$ into the random function $g$ of the output S.P. $\left(U_{t}(g), t \in R\right)$. The condition 1.1(i) states that the flow of time does not affect the filter operation; thus 1.1(i) is the mathematical expression of what is termed timeinvariance in the engineering literature. The condition 1.1(ii), which we shall call the subordination property of $T$ (cf. \$1), subsumes what is referred to as the causal or non anticipative property of the filter, viz., the filter response at any moment depends on inputs fed into it in the past alone. In particular, the response of a linear filter is a linear combination of past inputs or a limit thereof; thus

$$
\text { for each } x \in \mathscr{D}_{T}, \quad T(x) \in \subseteq\left(U_{t}(x), t \leqq 0\right) \subseteq S_{x}{ }^{3}
$$

In short, the requirements (1.1(i), (ii) are satisfied by all time-invariant, causal, linear filters. As for 1.1(iii), it is the natural assumption to make in order to get a decent mathematical theory.

In the engineering literature non-rigorous methods are used to obtain a function $\phi$ on $R$ such that $G^{\prime}(\lambda)=|\phi(\lambda)|^{2} F^{\prime}(\lambda)$, where $F^{\prime}, G^{\prime}$ are the spectral densities of the input and output S.P.'s. $\phi$ is called the frequency-response function. But to assert that such a $\phi$ exists is tantamount to asserting that $T=\int_{-\infty}^{\infty} \phi(\lambda) E(d \lambda)$. Thus, Theorem 1.1 provides a rigorous basis for the introduction of the frequency-response function into the theory. We thereby extend the work of Youla, Castriota, and Carlin [5] on the rigorous development of the classical (nonstatistical) theory of passive filters.

3. The commutant; logical order of results. From 1.1(i), (ii) we can show that $\mathfrak{H}_{0}$ reduces $E$ and that $T$ lives on $\mathfrak{H}_{0}$, i.e., $\mathscr{D}_{T} \cup \mathfrak{R}_{T} \subseteq \mathcal{H}_{0}$. Hence, without loss of generality, we can treat $\mathfrak{H C}_{0}$ as our overall Hilbert space, and suppose that $U_{t}$ and $E(B)$ are defined merely on $\mathfrak{F}_{0}$. In short, we can take $\mathfrak{F}_{0}=\mathfrak{H C}$. From here on we shall therefore remove the hypothesis 1.1 (iii) in favor of the following

3.1. Assumption. $T$ is closed, $\mathscr{D}_{T}$ is everywhere dense in $\mathfrak{H C}$, and $\mathfrak{H C}$ is separable.

Given that 3.1 holds, the well-known necessary and sufficient condition that $T=\int_{-\infty}^{\infty} \phi(\lambda) E(d \lambda)$ is in terms of the operator $\mathrm{H}$ $=\int_{-\infty}^{\infty} \lambda E(d \lambda)$ :

$B$ is bounded and $B H \subseteq B B \Rightarrow B T \subseteq T B$;

: $S(A)$ denotes the (closed) subspace spanned by $A$. 
briefly, $\{H\}^{\prime} \subseteq\{T\}^{\prime}$, where $F^{\prime}$ is the commutant of the family of operators $\mathfrak{F}^{4}$

Now $U_{t}$ and $H$ commute (being spectral integrals with respect to the same $E$ ), therefore by (3.2) $U_{t} T=T U_{t}, t \in R$. Next, the cyclic subspaces reduce all spectral integrals, therefore $L_{x} H \subseteq H L_{x}$ for $x \in \mathcal{K}$, whence 1.1(ii) follows easily. Thus the implication

$$
(3.2) \Rightarrow 1.1 \text { (i), (ii) }
$$

is trivial, and the well-known theorem involving (3.2) follows easily form Theorem 1.1. To establish Theorem 1.1 we have to prove, in effect, the converse implication

$$
1.1 \text { (i), (ii) } \Rightarrow(3.2) \text {. }
$$

This is much harder, since in 1.1(i) $T$ is required to commute with a much smaller class than in (3.2), and 1.1(ii) is not a commutation relation at all.

Actually, the use of cyclic projections in the usual proof of the theorem involving the commutant condition (3.2), cf. [3, p. 351], renders the latter somewhat superfluous for our purposes. The most logical and economical order of proving propositions seems to be the following in which the theorem involving (3.2) comes last:

(3.3) 1.1(i), (ii) $\Rightarrow$ for each $x \in \mathfrak{N}_{T}, L_{x} T \subseteq T L_{x},{ }^{5}$ where $\mathfrak{N}_{T}=U_{\alpha \in D_{T}} S_{\alpha}$ is an (unclosed) linear manifold.

(3.4) 1.1(i), (ii) $\Leftarrow$ for each $x \in \mathbb{N}_{T}, L_{x} T \subseteq T L_{x}$.

(3.5) For each $x \in \mathfrak{M T}_{T}, L_{x} T \subseteq T L_{x} \Leftrightarrow T=\int_{-\infty}^{\infty} \phi(\lambda) E(d \lambda)$.

(3.6) $\{H\}^{\prime} \subseteq\{T\}^{\prime} \Leftrightarrow T=\int_{-\infty}^{\infty} \phi(\lambda) E(d \lambda)$.

4. Outline of the proof. The following three lemmas on cyclic subspaces and projections play an important role in our proof:

(4.1) If $x \in \mathcal{S}_{\alpha}$, then $\exists C_{x, \alpha} \in 囚$ such that $L_{x}=E\left(C_{x, \alpha}\right) L_{\alpha}$.

(4.2) The intersection of two cyclic subspaces is cyclic; in fact,

$$
S_{L_{\alpha \beta}(\alpha)}=S_{\alpha} \cap S_{\beta}=S_{L_{\alpha \beta}(\beta)},
$$

where $L_{\alpha \beta}$ is the projection onto $S_{\alpha} \cap S_{\beta}$.

(4.3) For $\alpha, \beta \in \mathfrak{H}$, if $\beta^{\prime}=\beta-L_{\alpha \beta}(\beta)$, then

$$
s_{\alpha}+s_{\beta}=s_{\alpha}+s_{\beta^{\prime}}, \quad s_{\alpha} \cap s_{\beta^{\prime}}=\{0\} .
$$

- This result is due to von Neumann, F. Riesz and Mimura, cf. e.g. [3, p. 351]. In [3] the $B$ in (3.2) is taken to be self-adjoint. But this is only a slight change, since every bounded operator has self-adjoint real and imaginary parts. Stone $[4$, p. 189] refers to a somewhat more general version of this result as the S.O.M. Theorem, after Sasaki, Ogasawara and Mimura.

- In other words, for each $x \in \mathbb{M T}_{T}, \mathrm{~S}_{x}$ reduces $T$. 
We also need several lemmas concerning the operator $T$ :

(4.4) The condition $T L_{x} \subseteq L_{x} T$ for $x \in \mathscr{M}_{T}$ (cf. (3.3)) is equivalent to the condition

$$
T(x) \in S_{x} \text { for } x \in D_{T} \text { and } L_{x}\left(D_{T}\right) \subseteq D_{T} \quad \text { for } \quad x \in \mathfrak{M}_{T} .
$$

(4.5) For a closed operator $T, U_{t} T=T U_{t}$ for $t \in R$, if and only if $E(B) T \subseteq T E(B)$ for $B \in 囚$.

(4.6) If $T$ is closed, $U_{t} T=T U_{t}$ for $t \in R$, and $\psi$ is bounded, then

$$
\left\{\int_{-\infty}^{\infty} \psi(\lambda) E(d \lambda)\right\}\left(D_{T}\right) \subseteq D_{T} .
$$

(4.7) If $T$ is as in (4.6), then

$$
\begin{aligned}
& L_{\alpha}(\beta) \in D_{T} \quad \text { for } \beta \in D_{T} \text { and } \alpha \in S_{\beta}, \\
& L_{\alpha \beta}(\beta) \in D_{T} \text { for } \alpha \in \mathcal{H} \text { and } \beta \in D_{T} \text {. }
\end{aligned}
$$

(4.8) If $T$ is closed and satisfies 1.1(i), (ii), then, for each $\alpha \in D_{T}$, there exists a spectral integral $R_{\alpha}$ such that

$$
\text { Rstr. } S_{\alpha} \cap D_{T} T=\text { Rstr. } \oint_{\alpha} \cap_{R_{\alpha}} R_{\alpha}{ }^{6}
$$

Finally, we need the following property of spectral integrals:

(4.9) If $S=\int_{-\infty}^{\infty} \psi(\lambda) E(d \lambda)$ is any spectral integral, $\alpha$ is any vector, and $[\alpha]=\left\{x: x \in \mathcal{H C} \& S_{x}=S_{\alpha}\right\}$, then $\mathfrak{D}_{S} \cap[\alpha]$ is an infinite set with cluster point $\alpha$; in fact,

$$
\alpha_{n}=\int_{-\infty}^{\infty} \min \{1, n /|\psi(\lambda)|\} E(d \lambda) \alpha \in D_{S} \cap[\alpha], \quad \text { and } \quad \alpha_{n} \rightarrow \alpha .
$$

Space does not permit us to say more concerning these lemmas, nor even to indicate the main steps in the proofs of (3.3)-(3.5). (3.3) and (3.5), of course, yield Theorem 1.1, from which (3.6) follows at once as indicated in $\$ 3$.

5. Corollaries. Let mult $E=1$. Then taking an $\alpha$ in (4.1) for which $S_{\alpha}=\mathfrak{H}$, we see that every cyclic projection $L_{x}$ is a spectral projection $E\left(C_{x}\right)$. Hence by (4.5) and (4.4) every time-invariant operator is subordinative. In this case Theorem 1.1 reduces to the following:

5.1. Corollary. When mult $E=1$, every closed, time-invariant, linear operator with an everywhere dense domain is a spectral integral. ${ }^{7}$

- This means, of course, that $S_{\alpha} \cap D_{T}=S_{\alpha} \cap D_{R_{\alpha}}$, and, for each $x$ in this set, $T(x)=R_{\alpha}(x)$.

7 In the special case $\mathcal{H}=L_{2}(-\infty, \infty),\left\{U_{t}(f)\right\}(\lambda)=f(t+\lambda)$, this reduces to a result first proved by Bochner with the restriction (now seen to be unnecessary) that $T$ is bounded [1]. 
Next let mult $E \leqq \aleph_{0}$, but $T$ be bounded on $H$. Then trivially $L_{x}\left(D_{T}\right) \subseteq D_{T}, x \in \mathcal{F}$. Hence by (4.4) and (3.5) every bounded subordinative operator is time-invariant. In this case Theorem 1.1 yields the following:

5.2. Corollary. Every linear, subordinative operator, which is bounded on $\mathfrak{F}$, is a spectral integral.

When mult $E>1$, Corollary 5.1 fails even for a bounded $T$ : just take $T=L_{\alpha}$, where $L_{\alpha}$ does not commute with some $L_{\beta}$. Next, when $T$ is closed but unbounded, Corollary 5.2 fails even when mult $E=1$. Take $T=i D$, where $D$ is the differentiation operator restricted to the absolutely continuous functions $f$ in $L_{2}(C)$, such that $f(1)=0, C=$ unit circle, and $U_{t}$ is translation: $\left\{U_{t}(f)\right\}\left(e^{i \theta}\right)=f\left\{e^{i(\theta+t)}\right\} . T$ is closed, subordinative and symmetric with an everywhere dense domain, but it is not self-adjoint and not therefore a spectral integral. These examples show that the hypotheses of Theorem 1.1 are in a sense the best possible.

Acknowledgment. The writer would like to thank Professor J. Feldman for his useful suggestion that time-invariance might follow from the requirement $L_{x} T \subseteq T L_{x}$ (cf. (3.4)). He would also like to thank Drs. I. W. Sandburg and V. E. Benes of the Bell Telephone Laboratories for stimulating conversations on the subject.

\section{REFERENCES}

1. S. Bochner, Ein Satz über lineare Operationen, Math. Z. 29 (1929), 737-743.

2. A. N. Kolmogorov, Stationary sequences in Hilbert space, Byull. Moskov. Gos. Univ. Mat. 2 (1941), no. 6. (Russian)

3. F. Riesz and B. Sz.-Nagy, Functional analysis, Ungar, New York, 1959.

4. M. H. Stone, On unbounded operators in Hilbert space, J. Indian Math. Soc. 16 (1951), 155-192.

5. D. C. Youla, L. J. Castriota and H. J. Carlin, Bounded real scattering matrices and the foundations of linear passive network theory, IRE Trans. Circuit Theory CT-6 (1959), 102-124.

\section{INDIANA UNIVERSITY}

